Editorial

\title{
Acknowledgement to Reviewers of Economies in 2018
}

\section{Economies Editorial Office}

MDPI, St. Alban-Anlage 66, 4052 Basel, Switzerland

Published: 1 February 2019

Rigorous peer-review is the corner-stone of high-quality academic publishing. The editorial team greatly appreciates the reviewers who contributed their knowledge and expertise to the journal's editorial process over the past 12 months. In 2018, a total of 69 papers were published in the journal, with a median time to first decision of 32.48 days and a median time to publication of 94 days. The editors would like to express their sincere gratitude to the following reviewers for their cooperation and dedication in 2018:

\author{
Abbas, Azhar \\ Abdel-Latif, Hany \\ Achim, Monica Violeta \\ Adem, Teferi \\ Adu-Gyamfi, Samuel \\ Affuso, Ermanno \\ Ahmad, Ahmad Hassan \\ Akobirshoev, Ilhom \\ Albani, Viviana \\ Aleluia Da Silva Reis, Lara \\ Alina, Haller \\ Alonso-Villar, Olga \\ Ammi, Chantal \\ Andersson, Fredrik N.G. \\ Angeon, Valérie \\ Anwar, Sajid \\ Arias, Xosé Carlos \\ Aristizabal, Geovanny Castro \\ Aristovnik, Aleksander \\ Artavanis, Nikolaos \\ Arvanitidis, Paschalis \\ Asongu, Simplice \\ Atukeren, Erdal \\ Baek, Jungho \\ Baek, Seungho \\ Baeza, M. A. \\ Bandyopadhyay, Siddhartha \\ Basu, Arnab \\ Bayer, Patrick \\ Beans, Julie \\ Benito, Bernardino \\ Benjamin, Dave \\ Bilan, Irina \\ Bilas, Vlatka \\ Binder, Carola
}

\author{
Bjornlund, Henning \\ Blazsek, Szabolcs \\ Borochkin, Alexander \\ Boudreaux, Christopher \\ Breetz, Hanna \\ Brem, Alexander \\ Britzelmaier, Bernd \\ Butkus, Mindaugas \\ CAI, Jun \\ Calin, Adrian Cantemir \\ Caporale, Guglielmo Maria \\ Caraiani, Petre \\ Carmody, Pádraig \\ Caruso, Gianluca \\ Cassou, Steven P. \\ Cebula, Richard \\ Ceptureanu, Eduard Gabriel \\ Chan, Leo $\mathrm{H}$. \\ Chang, Tsangyao \\ Chileshe, Nicholas \\ Choi, Yongrok \\ Chowdhury, Murshed \\ Costa Freitas, Maria De Belém \\ Costantini, Mauro \\ Crespo, Nuno \\ Cude, Brenda \\ Curea-Pitorac, Ruxandra Ioana \\ Curtis, Chadwick C. \\ Czudaj, Robert \\ Dahl, Roy Endre \\ Dal Bianco, Silvia \\ Dapice, David \\ Davidson, Sinclair \\ De Pace, Pierangelo \\ Dionísio, Andreia
}


Doucek, Petr

Drabkova, Zita

Drachal, Krzysztof

Dreger, Christian

Dreyer, Marc

Dungey, Mardi

Dutta, Nabamita

Eita, Joel Hinaunye

Ejdys, Joanna

Ekanayake, E. M.

Emmerling, Johannes

Erokhin, Vasilii

Ezebilo, Eugene E.

Färber, Gisela

Faria, Joao Ricardo

Farzanegan, Mohammad Reza

Ferreira, Candida

Firtescu, Bogdan

Flach, Lisandra

Forest, James

Franc, Sanja

Fuentes, Rosana

Gadea, Maria Dolores

Galanis, Giorgos

Ganau, Roberto

Garcia, Maria Teresa Medeiros

Garza-Rodriguez, Jorge

Gatto, Andrea

Gheorghe, Popescu H.

Gil-Alana, Luis

Gilbert, Stanley W

Gillman, Max

Giuliani, Diego

Glabadanidis, Paskalis

Głodowska, Agnieszka

Green, Erik

Guaita Martínez, José Manuel

Guarini, Giulio

Guesmi, Khaled

Guimaraes, Paula

Halicioglu, Ferda

Hall, Joshua

Hasanov, Fakhri

Hassan, Rohail

Hatzvi, Eden

Haughton, Jonathan

Hedvičáková, Martina

Heine, Dirk

Hillman, Arye L.

Hoang, Edward C.

Hoffmann, Roman

Holcombe, Randall
Ibragimov, Rustam

Ibrahim, Muazu

Isaksson, Ann-Sofie

Ishii, Hokuto

Ivanyna, Maksym

Iyke, Bernard Njindan

Jackson, Karen

Jacobsen, Joyce

Jankowska-Mihułowicz, Marzena

Jankowski, Michael

Janovec, Michal

Jennings, Colin

Jianu, Iulia

Jiménez, Alfredo

Jurdi, Doureige

Kallioras, Dimitrios

Kalulu, Mavuto

Kapri, Kul

Katusiime, Lorna

Khajeheian, Datis

Kharrazi, Ali

Kim, Euijune

Kiseláková, Dana

Kokabisaghi, Somayeh

Kopkin, Nolan

Koutmos, Dimitrios

Krzos, Grzegorz

Lahr, Michael

Laspa, Christina

Laurila, Hannu

Ławińska, Olga

Leblois, A.

Leite, Duarte Nuno

Lemi, Adugna

Lennard, Jason

Leonidas, Paroussos

Liebenehm, Sabine

Lin, Tin-Chun

liyama, Miyuki

López-Gutiérrez, Carlos

Lorde, Troy

Lorente, Daniel Balsalobre

Ludwig, Markus

Macuda, Małgorzata

Magazzino, Cosimo

Maier, Dorin

Maitah, Mansoor

Małgorzata, Markowska

Malhotra, D. K.

Mamatzakis, Emmanuel

Marcu, Nicu

Martinez, Felipe 
Martínez-Sánchez, Ángel

Mary, Sébastien

Maryska, Milos

Maswana, Jean-Claude

Matthews, Kent

Matuzeviciute, Kristina

McCord, Michael

McMillan, David

Mention, Anne-Laure

Michalski, Grzegorz

Mihai, Costica

Mikušková, Eva Ballová

Miller, Stephen

Miron, Dumitru

Mishra, Tapas

Mohelská, Hana

Moldovan, Octavian

Morley, Bruce

Morrissey, Oliver

Müller, Julian Marius

Munger, Michael C.

Murzin, Anton

Naumann, Elias

Nemec, Juraj

Newaz, Mohammad

Nguyen, Moon

Nguyen, T.Thanh-Binh

Nicolau, Juan Luis

Nisticò, Rosanna

Novickytė, Lina

Odusola, Ayodele

Ongan, Serdar

Osambela, Emilio

Owusu, Erasmus L.

Ozay, Ozge

Paradies, Yin

Pavlik, Jamie

Paz, Lourenco

Pederson, JoEllen G.

Peinado, Patricia

Perles-Ribes, José

Peters, Greg

Peters, Sanjay V.

Pfaffermayr, Michael

Piketty, Marie-Gabrielle

Pons, Vincent

Potrafke, Niklas

Privitera, Donatella

$\mathrm{Pu}$, Xujin

Quartey, Ebo Tawiah

Rafaj, Peter

Rakowski, David
Ratnayake, R.M. Chandima

Rawski, Thomas G.

Riaz, Umair

Rjoub, Husam

Robertson, Peter John

Robinson, Derrick

Romanelli, Mauro

Romano, Simone

Royer, Jeffrey S.

Rülke, Jan-Christoph

Ryder, Nicholas

Sadka, Gil

Saha, Sujata

Sakyi, Daniel

Salvia, Rosanna

Saucedo Delgado, Odra Angélica

Scaramozzino, Pasquale

Scholtens, Bert

Schwarz, Jiří

Scicchitano, Sergio

Sequeira, Sandra

Shahbaz, Muhammad

Siad, Si Mokrane

Signorelli, Marcello

Simkova, Martina

Škrinjarić, Tihana

Skryl, Tatiana

Snudden, Stephen

Sołoducho-Pelc, Letycja M.

Sonora, Robert

Spacek, Miroslav

Ssozi, John

Staatz, John M.

Stauvermann, Peter Josef

Stauvermann, Peter Josef

Stawska, Joanna

Stejskal, Jan

Stirzaker, Richard

Stöckl, Albert

Streimikiene, Dalia

Struk, Michal

Sudzina, Frantisek

Sundqvist, Jan-Olov

Svobodová, Libuše

Tabata, Ken

Tamura, Robert

Tan, Aaron

Tang, Leilei

Telg, Sean

Tilley, Elizabeth

Tiron-Tudor, Adriana

Tselios, Vassilis 
Tsopanakis, Andreas

Tvaronavičienė, Manuela

Tzeremes, Nickolaos G.

Upadhyaya, Kamal

Valaskova, Katarina

Van Den Broeck, Goedele

Vandeleene, Audrey

Visvizi, Anna

Vlachos, Dimitrios

Voica, Marian Catalin

Vokoun, Marek

Von Zur Muehlen, Peter

Walter, Stefan

Weber, Christoph S.

Wei, Taoyuan
$\mathrm{Wu}$, Tsaur-Chin

Wunnava, Phanindra

Yalamova, Rossitsa

Yang, Sheng

Yi, John C.

Yildiz, Özgür

Yonk, Ryan

Yoon, SunMan

Zagaglia, Paolo

Zaremba, Adam

Zhang, Ren

Zhou, Qian

Ziemba, Paweł

Zygmunt, Aleksandra

(C) 2019 by the author. Licensee MDPI, Basel, Switzerland. This article is an open access article distributed under the terms and conditions of the Creative Commons Attribution (CC BY) license (http://creativecommons.org/licenses/by/4.0/). 